\title{
Measuring the Kuroshio Current with ocean acoustic tomography
}

\author{
Naokazu Taniguchi ${ }^{\text {a) }}$ \\ Graduate School of Engineering, Hiroshima University, Hiroshima 739-8527, Japan \\ Chen-Fen Huang ${ }^{\text {b) }}$ \\ Institute of Oceanography, National Taiwan University, Taipei 106, Taiwan \\ Arata Kaneko \\ Graduate School of Engineering, Hiroshima University, Hiroshima 739-8527, Japan
}

Cho-Teng Liu

Institute of Oceanography, National Taiwan University, Taipei 106, Taiwan

Bruce M. Howe

Department of Ocean and Research Engineering, University of Hawai' i at Manoa, Honolulu, Hawaii 96822

Yu-Huai Wang

Institute of Applied Marine Physics and Undersea Technology, National Sun Yat-sen University, Kaohsiung 804, Taiwan

Yih Yang

Taiwan Ocean Research Institute, Kaohsiung 852, Taiwan

Ju Lin

Department of Physics, Ocean University of China, Qingdao 266100, China

Xiao-Hua Zhu

State Key Laboratory of Satellite Ocean Environment Dynamics, Second Institute of Oceanography, State Oceanic Administration, Zhejiang 310012, China

\section{Noriaki Gohda}

Aqua Environmental Monitoring Limited Liability Partnership, Mihara 723-0047, Japan

(Received 17 August 2012; revised 21 February 2013; accepted 29 April 2013)

Ocean current profiling using ocean acoustic tomography (OAT) was conducted in the Kuroshio Current southeast of Taiwan from August 20 to September 15, 2009. Sound pulses were transmitted reciprocally between two acoustic stations placed near the underwater sound channel axis and separated by $48 \mathrm{~km}$. Based on the result of ray simulation, the received signals are divided into multiple ray groups because it is difficult to resolve the ray arrivals for individual rays. The average differential travel times from these ray groups are used to reconstruct the vertical profiles of currents. The currents are estimated with respect to the deepest water layer via two methods: An explicit solution and an inversion with regularization. The strong currents were confined to the upper $200 \mathrm{~m}$ and rapidly weakened toward $500 \mathrm{~m}$ in depth. Both methods give similar results and are consistent with shipboard acoustic Doppler current profiler results in the upper $150 \mathrm{~m}$. The observed temporal variation demonstrates a similar trend to the prediction from the Hybrid Coordinate Ocean Model.

(C) 2013 Acoustical Society of America. [http://dx.doi.org/10.1121/1.4818842]

PACS number(s): 43.30.Pc, 43.60.Pt [JAC]

Pages: $3272-3281$

\section{INTRODUCTION}

Ocean acoustic tomography (OAT) is a powerful tool that can estimate the structure of sound speed (mainly proportional to temperature) and current in the ocean (Munk et al., 1995). Other than coastal sea studies (e.g., Park and Kaneko, 2000), most previous experiments have been carried out in the deep ocean, characterized with a full structure of the underwater

\footnotetext{
${ }^{a}$ Present address: Institute of Oceanography, National Taiwan University, Taipei 106, Taiwan.

${ }^{b}$ Author to whom correspondence should be addressed. Electronic mail: chenfen@ntu.edu.tw
}

sound channel (Cornuelle et al., 1985; Cornuelle et al., 1993; Dushaw et al., 1995; Dushaw et al., 1994; Howe et al., 1987; Send et al., 1995; Worcester et al., 1985; Yuan et al., 1999). However, its application to shallower regions with a sound speed profile (SSP) decreasing toward the bottom from the surface was limited because of the difficulty of ray resolvability (DeFerrari and Nguyen, 1986). The same reason leads to its infrequent application to the western boundary current regions located along the continental slopes.

The OAT is proposed as a technology to make longterm measurements of overlying currents because the deployed systems are compact, near the seafloor, and robust to activities of fishing and shipping, in contrast to the more 
complicated and expensive point moorings. As demonstrated in the 2008 Luzon Strait experiment (Taniguchi et al., 2010), an OAT system, placed near the underwater sound channel axis where only its upper half is constructed, provides an efficient method to measure the full-depth profile of current in the Luzon Strait with the Kuroshio intrusion and strong internal tide activity. Due to the complicated bottom topography and the oceanographic variability, the deep-water paradigm of stable, identifiable ray travel times is not available. Instead of identifying each ray, a three-ray group method is proposed using averaged arrival times for multiple peaks within each of the groups to reconstruct the vertical profiles of current.

In this study, we apply the strategy employed by the 2008 Luzon Strait experiment to an analysis of acoustic tomographic data obtained in the Kuroshio southeast of Taiwan in 2009. The emphasis is on determining the time-dependent current profile above the sound channel axis that is near the bottom. The paper is organized as follows: Sec. II provides a brief description of the 2009 Kuroshio tomographic experiment. Section III develops the formulation of the tomography problem with clock correction. Section IV outlines the ray group method used to reconstruct the current profiles. The inversion results and discussion are presented in Secs. V and VI, respectively. Finally, a few concluding remarks are made in Sec. VII.

\section{EXPERIMENT}

An OAT experiment composed of two acoustic stations (T1 and T2) separated by about $48 \mathrm{~km}$ was carried out in the Kuroshio southeast of Taiwan from August 20 to September 15, 2009 (Fig. 1). The seafloor depth ranges from 900 to $1300 \mathrm{~m}$ along the section between the tomographic stations. The precise distance between T1 and T2 $(48.069 \mathrm{~km})$ is estimated from the deployment GPS position of the subsurface moorings. The horizontal and vertical movement of the

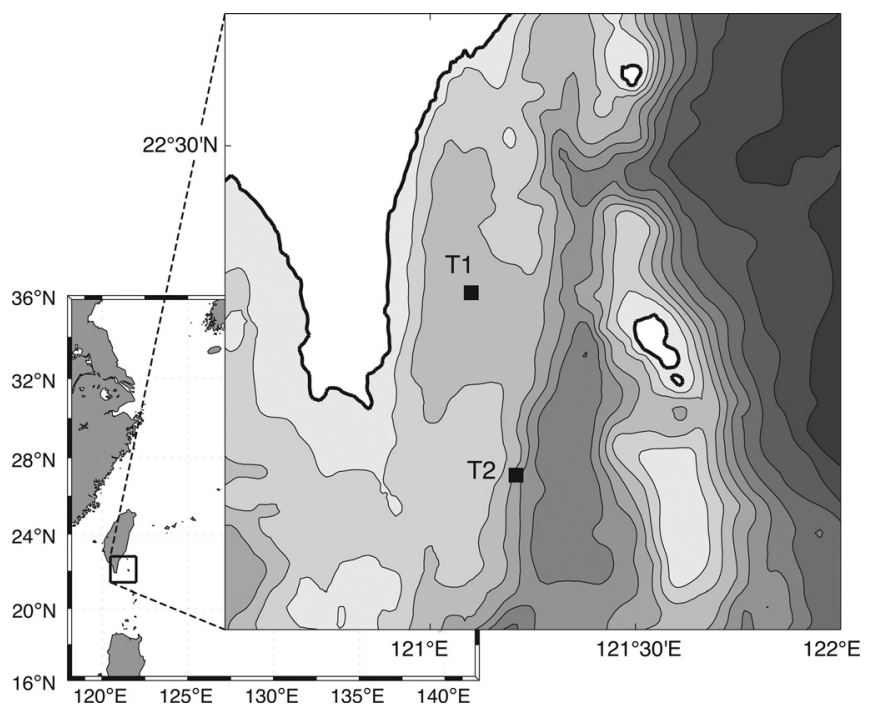

FIG. 1. Location maps of the observation site. The bathymetric chart around the observation site is shown in a magnified scale in the right side of the figure (contour interval is $500 \mathrm{~m}$ ). The square symbols indicate the locations of two mooring stations $\mathrm{T} 1$ and $\mathrm{T} 2$, each equipped with an OAT system. T1 was moored at $1243.5-\mathrm{m}$ depth near $22^{\circ} 10.343^{\prime} \mathrm{N}, 121^{\circ} 5.877^{\prime} \mathrm{E}$, and $\mathrm{T} 2$ was at $1330.3-\mathrm{m}$ depth near $21^{\circ} 45.049^{\prime} \mathrm{N}, 121^{\circ} 12.542^{\prime} \mathrm{E}$. Both OAT systems were suspended about $200 \mathrm{~m}$ above the seafloor. instrumentation on the short moorings was not measured because these movements have a small effect on the estimates of current velocities in this work.

At each acoustic station, T1 and T2, an $800 \mathrm{~Hz}$ narrowband organ pipe transducer with $50-\mathrm{Hz}$ bandwidth (after series tuning; Engineering Acoustics Inc.) was attached at a depth of approximately $1000 \mathrm{~m}$ to the mooring line, $200 \mathrm{~m}$ above the bottom. The source level of the transducer is about $13.2 \mathrm{~W}(182 \mathrm{~dB}$ re $1 \mu \mathrm{Pa}$ at $1 \mathrm{~m})$ and the electrical power consumption is $32 \mathrm{~W}$, much smaller than that used in conventional, longer-range and lower frequency tomographic experiments. The transmitted signal consisted of a phase modulated linear maximal pseudorandom sequence (referred to as an $\mathrm{M}$ sequence), to achieve adequate signal-to-noise ratio and high time resolution. One period of $\mathrm{M}$ sequence is transmitted, which reduces power consumption for longterm operation at the cost of a reduction in the SNR of received signals. The reduction of power consumption allows for smaller batteries, resulting in a compact system that is beneficial to multi-station tomography. This policy is justified from the experience of previous field work in which no serious problem occurs in the data analysis (Park and Kaneko, 2000; Taniguchi et al., 2010). The parameters of the acoustic signal are listed in Table I. Sound was transmitted from both the subsurface systems every $1.5 \mathrm{~h}, 10 \mathrm{~min}$ apart (not simultaneous due to the pulse length greater than the station-to-station distance as well as instrumentation constraints). Because our main focus is on measuring the Kuroshio Current, we apply a 5-day Hamming window to smooth the time series of differential travel times (described in the following text), which suppresses noise including travel time variations caused by internal tides and waves.

In this study, typically differential travel times are small, less than $7 \mathrm{~ms}$. Hence highly accurate clocks, such as an atomic oscillator, are desired for a subsurface OAT experiment where the system cannot receive GPS signals. However, when applied to a subsurface moored system, the power requirements of a conventional atomic oscillator result in a large electrical power consumption with corresponding large and expensive battery packs (Worcester et al., 1985). To address these issues, a hybrid clock system, composed of both a quartz and an atomic clock oscillator, is employed. Clock accuracy is maintained by correcting the frequency offset of the continuously running quartz clock by the intermittently operated atomic oscillator. Unfortunately in this experiment, the start timing of the clock system has some drift resulting from a mistaken hardware design, so any trend of time evolution of the first arrival peak is severely contaminated by the clock drift. Therefore the clock

TABLE I. Transmission signal parameters.

\begin{tabular}{lc}
\hline \hline Carrier frequency, $f_{0}$ & $800 \mathrm{~Hz}$ \\
Order of M-sequence & 12 \\
Q, cycles per digit & 12 \\
Digit duration & $15 \mathrm{~ms}$ \\
Sequence length & 4095 digits \\
Period duration & $61.425 \mathrm{~s}$ \\
Number of periods & 1 \\
\hline \hline
\end{tabular}


correction method proposed by Taniguchi et al. (2010) is applied here. In this method, currents for the overlying layers are calculated relative to the deepest layer. As a result, the travel times for rays passing through the upper layers are referenced to near axial rays. If current meter data are available in the deepest layer, the relative values of currents of the upper layers can be converted to absolute values.

The shipboard Acoustic Doppler Current Profiler (ADCP) (RDI $150 \mathrm{kHz}$ Ocean Surveyor) was used to collect data along path T1-T2 three times on August 19. The ADCP measured the ocean currents between 10 and $150 \mathrm{~m}$ below the sea surface. The raw ADCP velocities, acquired with a bin length of $8 \mathrm{~m}$, were processed using a phase-averaging method to remove tidal variations (Chang et al., 2008) and compared with the OAT data. On the mooring line T1, a current meter was attached $10 \mathrm{~m}$ above the transceiver. CTD casts were conducted at the acoustic stations, T1 and T2, during the deployment and recovery cruises to provide estimates of the sound speed fields.

\section{FORMULATION OF THE TOMOGRAPHY PROBLEM WITH CLOCK CORRECTION}

The total travel time for the $i$ th acoustic ray path is composed of the contributions from path segments in $N$ depth layers (counting from the deepest layer)

$$
\tau_{i}^{ \pm}=\sum_{j=1}^{N} \frac{l_{i j}}{C_{j} \pm u_{j}} \pm \tau_{\mathrm{err}}, \quad i=1,2, \ldots, M
$$

for a transmission in the positive/negative direction, respectively. $C_{j}$ and $u_{j}$ are the range-averaged sound speed and current for the $j$ th layer, respectively. $l_{i j}$ is the arc length traveled by the $i$ th ray in the $j$ th layer. $\tau_{\text {err }}$ is the relative clock error for the paired station (the difference between the absolute clock errors at each station) and is a constant for all rays. Notice here $j=1$ corresponds to the deepest layer. When calculating the differential travel time for the $i$ th ray [after linearizing Eq. (1) using $C_{j}^{2} \gg u_{j}^{2}$ ], the relative clock error still appears, with a factor of 2 , as shown below:

$$
\Delta \tau_{i}\left(=\tau_{i}^{+}-\tau_{i}^{-}\right)=-2 \sum_{j=1}^{N} \frac{l_{i j} u_{j}}{C_{j}^{2}}+2 \tau_{\mathrm{err}}, \quad i=1,2, \ldots, M .
$$

It can be expressed in a matrix form as

$$
\left[\begin{array}{c}
\Delta \tau_{1} \\
\Delta \tau_{2} \\
\vdots \\
\Delta \tau_{M}
\end{array}\right]=\left[\begin{array}{cccc}
\frac{-2 l_{11}}{C_{1}^{2}} & \frac{-2 l_{12}}{C_{2}^{2}} & \cdots & \frac{-2 l_{1 N}}{C_{N}^{2}} \\
\frac{-2 l_{21}}{C_{1}^{2}} & \frac{-2 l_{22}}{C_{2}^{2}} & \cdots & \frac{-2 l_{2 N}}{C_{N}^{2}} \\
\vdots & \vdots & \ddots & \vdots \\
\frac{-2 l_{M 1}}{C_{1}^{2}} & \frac{-2 l_{M 2}}{C_{2}^{2}} & \cdots & \frac{-2 l_{M N}}{C_{N}^{2}}
\end{array}\right]\left[\begin{array}{c}
u_{1} \\
u_{2} \\
\vdots \\
u_{N}
\end{array}\right]+\left[\begin{array}{c}
2 \tau_{\mathrm{err}} \\
2 \tau_{\mathrm{err}} \\
\vdots \\
2 \tau_{\mathrm{err}}
\end{array}\right]
$$

The term $\tau_{\text {err }}$ can be eliminated by subtracting the differential travel time of a specific ray from those of all rays. Notice that the equation subtracted from itself becomes $0=0$, which is indefinite. If other independent information of current is available (i.e., the current in a specific layer), this information can be used to replace the indefinite equation. For example, if the first (deepest) ray is selected as a reference and some current information for the first layer (referred to as $\left.u_{\mathrm{ref}}\right)$ is given, the differential travel times relative to the first ray $\left(\Delta \tau_{21}, \Delta \tau_{31}, \ldots, \Delta \tau_{M 1}\right)$ may be expressed as

$$
\left[\begin{array}{c}
u_{\mathrm{ref}} \\
\Delta \tau_{21} \\
\vdots \\
\Delta \tau_{M 1}
\end{array}\right]=\left[\begin{array}{cccc}
1 & 0 & \cdots & 0 \\
\frac{-2\left(l_{21}-l_{11}\right)}{C_{1}^{2}} & \frac{-2\left(l_{22}-l_{12}\right)}{C_{2}^{2}} & \cdots & \frac{-2\left(l_{2 N}-l_{1 N}\right)}{C_{N}^{2}} \\
\vdots & \vdots & \ddots & \vdots \\
\frac{-2\left(l_{M 1}-l_{11}\right)}{C_{1}^{2}} & \frac{-2\left(l_{M 2}-l_{12}\right)}{C_{2}^{2}} & \cdots & \frac{-2\left(l_{M N}-l_{1 N}\right)}{C_{N}^{2}}
\end{array}\right]\left[\begin{array}{c}
u_{1} \\
u_{2} \\
\vdots \\
u_{N}
\end{array}\right] .
$$

There are two ways to specify $u_{\text {ref. }}$. The first is to set it to zero and then the currents of overlying layers are determined relative to the first layer. The other way is to use the current meter data $\left(u_{\mathrm{CM}}\right)$ as $u_{\text {ref }}$. In this case, the reconstructed overlying currents are absolute currents.

Equation (4) can be reduced to the following form:

$$
\mathbf{y}=\mathbf{E x}+\mathbf{n},
$$

where $\mathbf{y}$ is the data vector, $\mathbf{E}$ the observation matrix, and $\mathbf{x}$ the unknown variable model vector containing the layer currents. We have introduced a residual vector $\mathbf{n}$ to represent the error terms. Typically, the residual vector is measurement noise but here the interpretation of $\mathbf{n}$ is broadened to include modeling errors.

Two methods are used to reconstruct the vertical profiles of overlying currents: An explicit solution and an inversion 
accompanied by a regularization technique. Both methods are described in the following text.

\section{A. Explicit solution}

The tomographic domain is divided into $N$ depth layers, where $N$ is chosen by considering the ray simulation results described in Sec. IV. We define ray groups in the following way. The first ray group travels through only the first (deepest) depth layer, and the second ray group travels through both the first and second depth layers, and so on, resulting in the number of ray groups $M$ equal to the number of depth layers $N$ (in this case $M=N=3$ ).

In this evenly determined case, the observation matrix $\mathbf{E}$ becomes a lower triangular matrix and an explicit solution can be obtained directly in the following sequential form:

$$
\begin{aligned}
u_{1}= & u_{\mathrm{ref}}, \\
u_{2}= & -\frac{C_{2}^{2}}{2 l_{22}}\left\{\Delta \tau_{21}+\frac{2\left(l_{21}-l_{11}\right)}{C_{1}^{2}} u_{1}\right\}, \\
u_{3}= & -\frac{C_{3}^{2}}{2 l_{33}}\left\{\Delta \tau_{31}+\frac{2\left(l_{31}-l_{11}\right)}{C_{1}^{2}} u_{1}+\frac{2 l_{32}}{C_{2}^{2}} u_{2}\right\}, \\
& \vdots \\
u_{N}= & -\frac{C_{N}^{2}}{2 l_{M N}}\left\{\Delta \tau_{M 1}+\frac{2\left(l_{M 1}-l_{11}\right)}{C_{1}^{2}} u_{1}+\frac{2 l_{M 2}}{C_{2}^{2}} u_{2}\right. \\
& \left.+\cdots+\frac{2 l_{M N-1}}{C_{N-1}^{2}} u_{N-1}\right\},
\end{aligned}
$$

where $u_{\text {ref }}$ is the reference velocity in the first depth layer and $l_{1 j}=0$ for the $j$ values except $j=1$.
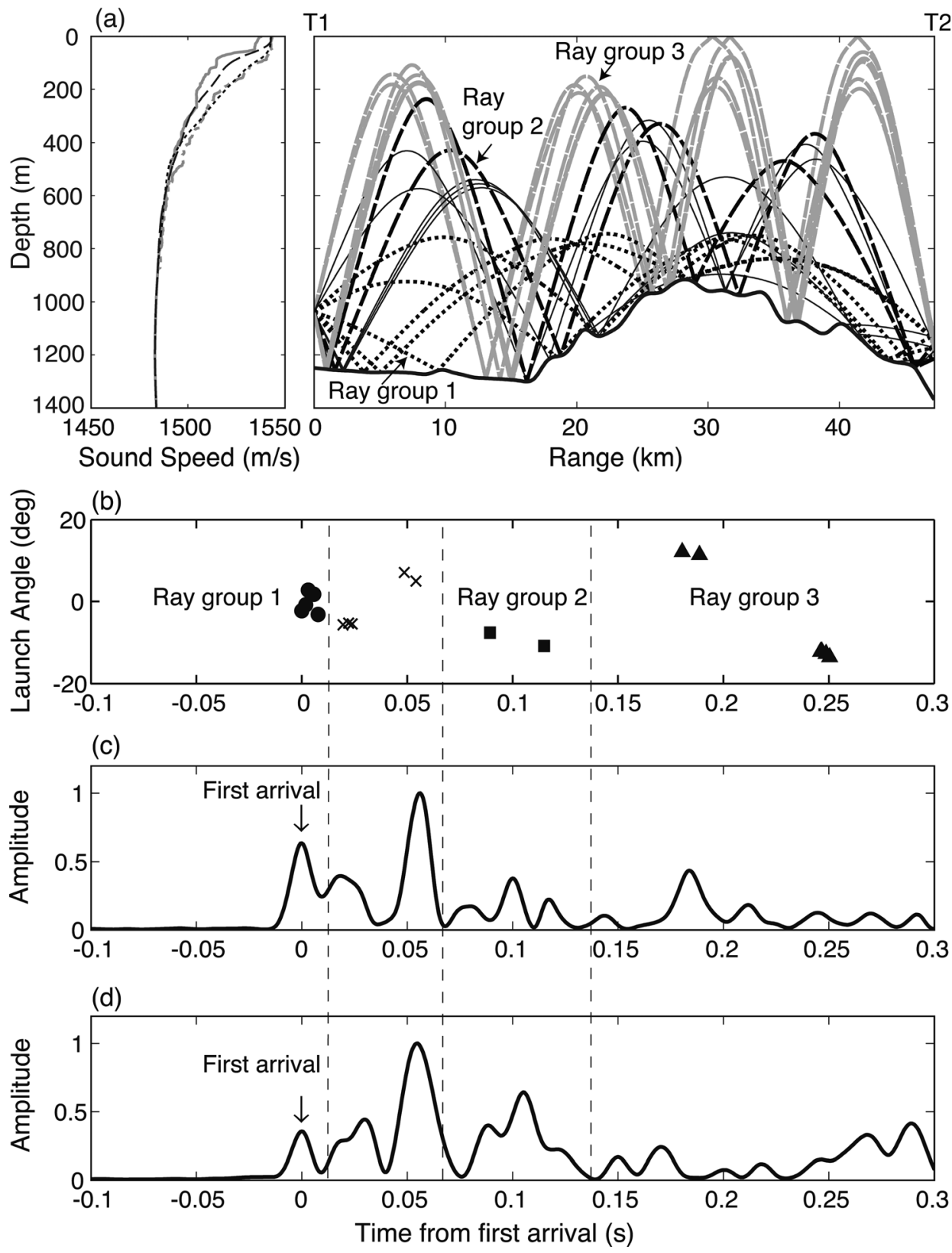

FIG. 2. Ray simulation and typical observed arrival patterns. Four sound speed profiles are shown in the left panel of (a): Two are the temporal average of the Hybrid Coordinate Ocean Model (HYCOM) predictions and the other two are from CTD measurements, both are at $\mathrm{T} 1$ and $\mathrm{T} 2$, respectively. The results of the rangedependent ray simulation are shown with (a) the ray pattern and (b) the travel time versus launch angle plot. Typical examples of the reciprocal arrival patterns observed at (c) $\mathrm{T} 1$ and (d) T2 are also shown. In the right panel of (a), the dotted, dark dashed, and gray dashed lines indicate ray groups 1, 2, and 3, respectively. In (b), the circle, square, and triangle symbols indicate ray groups 1,2 , and 3 , respectively. Individual ray groups are bounded with the dashed lines. 


\section{B. Inversion with regularization}

The inversion with regularization method overcomes certain limitations of the explicit method. Specifically, the explicit method requires that the number of ray groups equals the number of depth layers, and the solution is degraded due to the propagation of errors. For such cases, the inversion method is preferable, yielding a smoother solution by regularization (Rajan et al., 1987).

The cost function $J$ consists of the data misfit and smoothness measure of solution vector $\mathbf{x}$,

$$
J=\mathbf{n}^{T} \mathbf{n}+\lambda \mathbf{x}^{T} \mathbf{L}^{T} \mathbf{L} \mathbf{x}
$$

where $\lambda$ is a Lagrange multiplier and superscript $T$ denotes the transpose of the matrix. $\mathbf{L}$ is the second derivative operator. This smoothness measure penalizes solutions that are rough in a second derivative sense.

By minimizing $J$, the expected solution $\tilde{\mathbf{x}}$ is given by

$$
\tilde{\mathbf{x}}=\left(\mathbf{E}^{T} \mathbf{E}+\lambda \mathbf{L}^{T} \mathbf{L}\right)^{-1} \mathbf{E}^{T} \mathbf{y} .
$$

$\lambda$ is so chosen that the residual defined by $\|\tilde{\mathbf{n}}\|^{2}=\|\mathbf{y}-\mathbf{E} \tilde{x}\|^{2}$ is less than a pre-determined value of $1(\mathrm{~ms})^{2}$ (corresponding to $2.5 \mathrm{~cm} / \mathrm{s}$ for a $50-\mathrm{km}$ range).

For this inversion method, the solution uncertainty $\mathbf{P}$ may be expressed by (Wunsch, 1996)

$$
\mathbf{P}=\left(\mathbf{E}^{T} \mathbf{E}+\lambda \mathbf{L}^{T} \mathbf{L}\right)^{-1} .
$$

\section{RAY SIMULATION AND GROUPING}

Range-dependent ray simulation is used to determine the observation matrix $\mathbf{E}$ in Eq. (5). The experimental geometry is shown in Fig. 2(a). The bathymetry obtained from a separate multi-beam survey with a horizontal grid size length of $100 \mathrm{~m}$ (Liu et al., 1998) is used as the bottom topography data. The temperature and salinity data supplied from the HYCOM predictions (Chassignet et al., 2009) are used to construct the reference sound speed field(s) for the ray simulation. Six equally spaced temporally average profiles from August 3 to September 30, 2009, are determined along the $48 \mathrm{~km}$ transmission line. The sound speed profiles estimated from the conductivity-temperature-depth (CTD) casts (solid line for $\mathrm{T} 1$ and dashed-dotted line for T2) and the HYCOM predictions (dashed line for $\mathrm{T} 1$ and dotted line for T2) are in good agreement. The sound speed is slower at the north station (T1) than at the south station (T2), implying that water is cooler toward the north. Figure 2 shows the simulated ray diagram and travel time distribution together with typical snapshots of measured arrival patterns during the experiment.

The sound speed and its gradient determine the path length and travel time of rays between two stations. The simulated rays are divided into three groups by considering the depths of the upper turning points. Rays that travel through the lower water layer experience weak refraction and bottom reflection, have shorter path lengths, and arrive at the receiver first [Fig. 2(a)]. Rays that travel through the middle and upper layers experience more bottom and surface reflections. The first and second group rays are called conventionally refracted bottom-reflected (RBR) rays and the third group ray surface-reflected bottom-reflected (SRBR) rays. The simulated travel times are plotted against the launch angles [Fig. 2(b)]. The multiple observed peaks in the arrival patterns [Figs. 2(c) and 2(d)] scatter around the simulated travel times. The ray path lengths used in the subsequent inversion are determined by averaging the path lengths of all rays in each ray group.

To investigate the effects of sound speed and current variabilities on reciprocal transmissions, a series of received arrival patterns is simulated using the same geometry as the experiment and the oceanographic conditions provided by the daily HYCOM prediction. For simplicity, the current effect is accounted for by adding or subtracting the current velocity (as a scalar quantity) from the actual sound speed in the ray tracing program. For each day, the arrival times and amplitudes of eigenrays (channel impulse response) are computed using the ray tracing program BELLHOP (Porter, 2011). Then a received arrival pattern is obtained by the convolution of the transmitted signal waveform with the impulse response of the channel. Note that we do not intend to fit the measured arrival patterns with the simulations but to qualitatively understand the travel time variability.

Figure 3 shows the effects of sound speed and current variability on the simulated acoustic arrival patterns. These arrival patterns are the results of overlapping and grouping of multipath eigenray arrivals. The symbols superimposed on the arrival pattern indicate different ray groups defined via the method described in the next subsection. Arrival times of first peaks (indicated by circles) are modulated by the sound speed near the bottom water. Compared with the ray simulation using the reference SSPs [Fig. 2(b)], the second group of arrivals (squares) is formed by not only the RBR rays but sometimes those that follow the steepest SRBR paths. However, we are not concerned with these

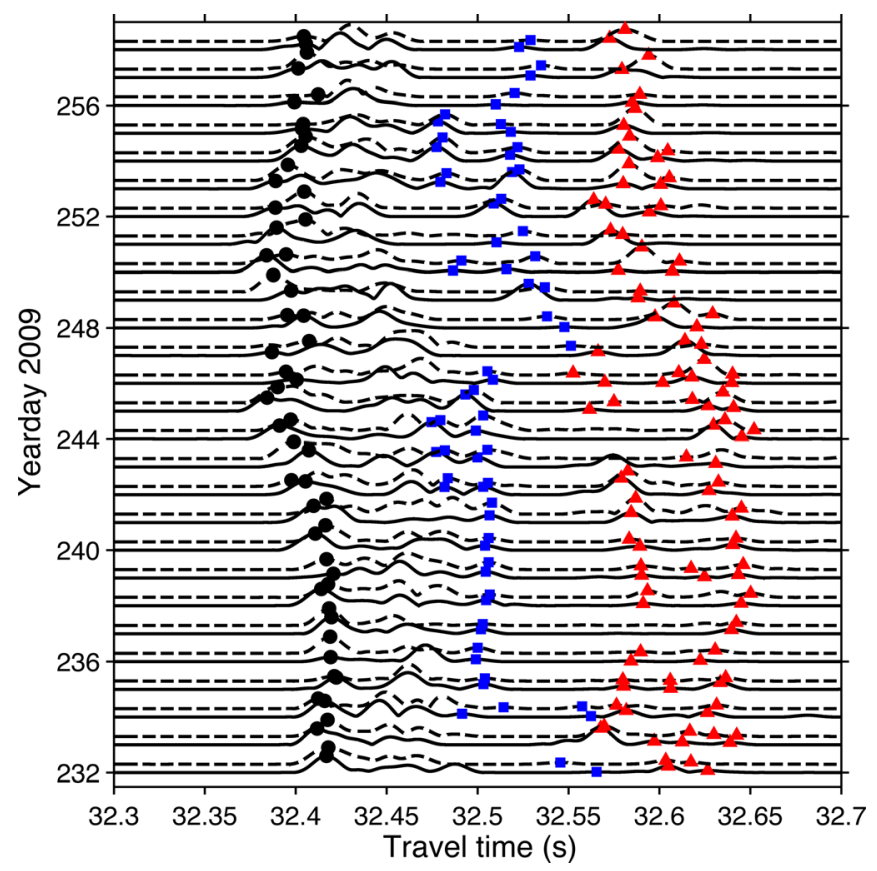

FIG. 3. (Color online) Simulated daily arrival patterns at T1 (solid line) and T2 (dashed line) from August 20 (yearday 232) to September 15, 2009. The peaks that belong to ray groups 1,2 , and 3 are indicated by circle, square, and triangle symbols, respectively. 
SRBR rays due to their small contribution (weak amplitude) to the arrivals. The third ray group (triangles) is formed by the combination of steepest RBR rays and gradual SRBR rays. Note that no threshold is used to pick up the above peaks because no noise is added to the synthetic data. The overall features of ray groups do not differ significantly from the reference environment.

\section{A. Data analysis: Ray grouping}

Significant peaks are identified in the arrival patterns of $\mathrm{T} 1$ and $\mathrm{T} 2$ as those with SNRs greater than $12 \mathrm{~dB}$. Ideally pairs of reciprocal arrival peaks for $\mathrm{T} 1$ and $\mathrm{T} 2$ are required for the calculation of differential travel times, and thus pathaveraged currents, but in this case, this is not possible because of the observed variability in the number, amplitude, and travel times of the peaks. This variability is likely a combination of factors including nonsimultaneity of transmissions, bottom interactions, and non-reciprocity due to current shear. Instead of identifying each ray, we propose a method of grouping and averaging for multiple arrival peaks referred to as the ray group method. Considering the distribution of ray paths from the surface to bottom, we shall here divide arrival peaks into three arrival groups and pairs of arrival peaks are searched for within each group. The first, second, and third ray groups (equivalent to the first, second, and third arrival groups, respectively) are selected to travel through only the lower layer $(>500 \mathrm{~m})$, the lower to middle layer $(>250 \mathrm{~m})$, and all three (lower to upper) layers, (a-1)

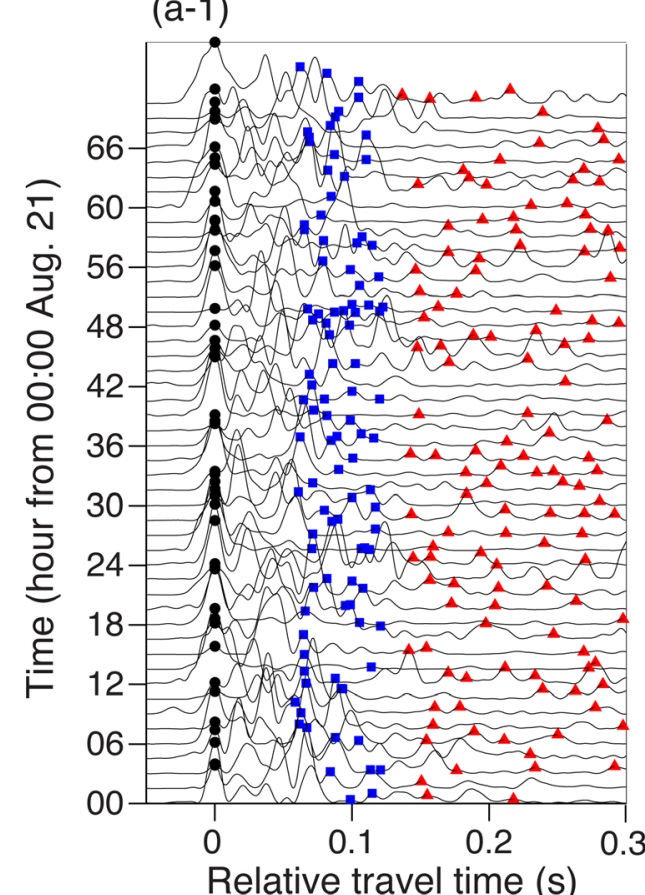

(b)

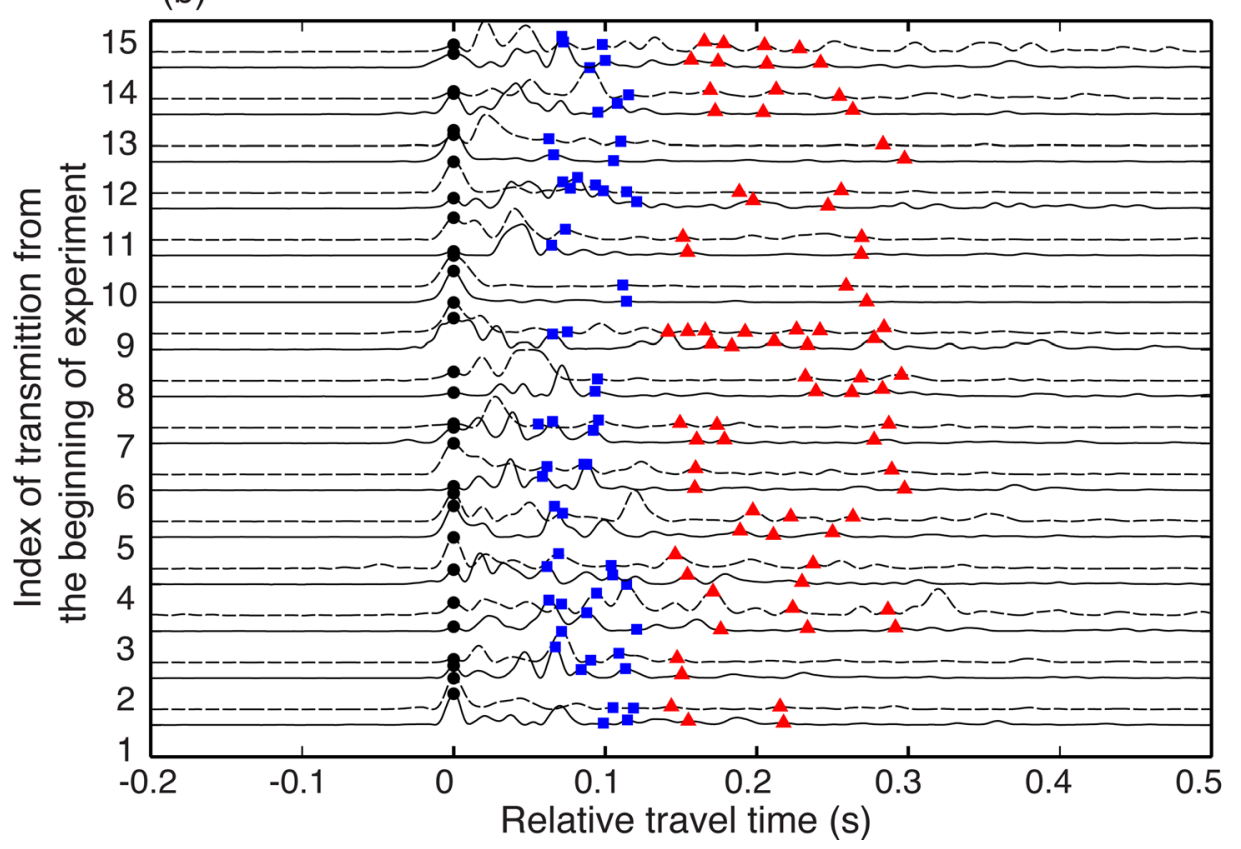

FIG. 4. (Color online) Measured arrival patterns stacked upward with time. Data received at $\mathrm{T} 1$ and $\mathrm{T} 2$ are plotted separately in (a) for the first 3 days and together in (b) for the first day. In (b), solid lines are used for T1 and dashed lines for T2 with some offset from T1. The circle, square, and triangle symbols are put on the peaks that belong to ray groups 1, 2, and 3, respectively. Note that the first arrival peak is aligned between transmissions. 
respectively. In the subsequent analysis, only the first arrival times are used as data of the first ray group. The second ray group has relative travel times from 70 to $140 \mathrm{~ms}$, measured from the first arrival time. The third ray group has relative travel times $140 \mathrm{~ms}$ or greater from the first arrival time. The arrival peaks of $\mathrm{T} 2$ that make pairs with those for $\mathrm{T} 1$ are searched for within the time delay of $2 \pm 15 \mathrm{~ms}$ for the second arrival group and $7 \pm 15 \mathrm{~ms}$ for the third arrival group. The mean difference times of 2 and $7 \mathrm{~ms}$ are obtained using the HYCOM predictions between $\mathrm{T} 1$ and $\mathrm{T} 2$, and the error bar $15 \mathrm{~ms}$ comes from one digit width of the $\mathrm{M}$ sequence. The selection of this time window is based on the time resolution for multiple peaks because the ray group method should generally be applicable even for cases that a priori information of current variations is not available.

Measured arrival patterns for the first 3 days are stacked upward as the time proceeds [Fig. 4(a)]. Different symbols are placed on those arrival peaks that satisfy the preceding condition for identifying a pair of significant arrival peaks: Circles for the first arrival peak, squares for the second arrival group, and triangles for the third arrival group. Both T1 and T2 data are compared in Fig. 4(b) (shown here are the first day only). The reciprocity of arrival patterns is not satisfied in some places, and the resulting peaks for $\mathrm{T} 1$ do not appear for $\mathrm{T} 2$. The differential travel times are determined by averaging all obtainable peaks within individual arrival groups. The identification errors of arrival peaks due to non-simultaneity of transmissions, bottom interactions, and non-reciprocity are much reduced through the grouping and averaging processes. In addition, the results are smoothed with the 5-day Hamming window to reduce noise.

\section{INVERSION RESULTS}

\section{A. Synthetic data}

To demonstrate the three-ray group method, the synthetic data shown in Fig. 3 are used as the input data for the inversion. First, the arrival peaks for each group are selected based upon the peak selection method proposed in the preceding text. Then the differential travel times are averaged from the obtainable peaks within the arrival groups.

Figure 5 shows the inversion results for the data on August 24 and September 11. The HYCOM predictions are used to set the current values near the bottom. Both explicit (solid line with circle) and regularization inversion (dashed line with square) methods are used to invert for the depth-averaged vertical current profile. The reconstructed profiles show good agreement in comparison with the HYCOM predictions (dotted line).

\section{B. Experimental data}

The currents in each layer determined from the explicit solution, Eq. (6), are shown in Fig. 6 with the time series plotted together with the differential travel times for ray groups 2 and 3 . Results for the two cases $u_{\text {ref }}=0$ and $u_{\text {ref }}=u_{\mathrm{CM}}$ are almost the same over the depth because $u_{\mathrm{CM}}$ is small. The reconstructed current decreases from $0.4 \mathrm{~m} / \mathrm{s}$ in the upper layer to $0.1 \mathrm{~m} / \mathrm{s}$ in the middle layer. The lower layer current, obtained by the deep current meter, varies in (a) Day 236

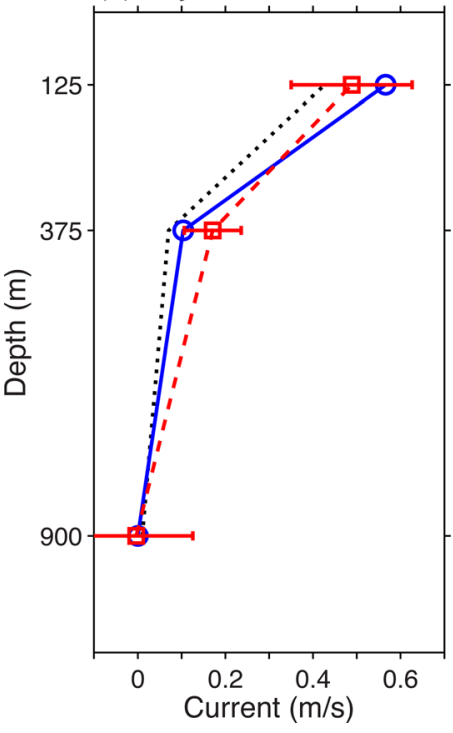

(b) Day 254

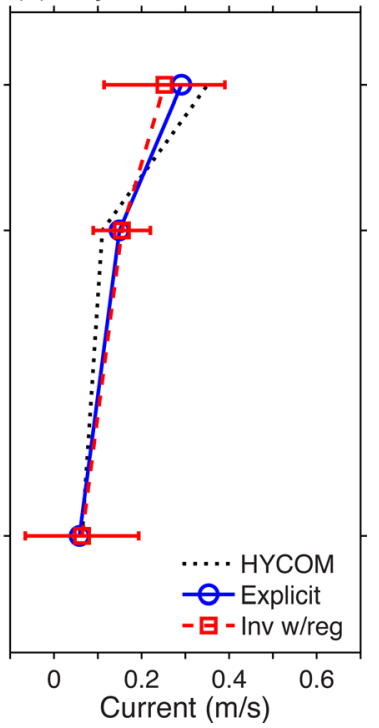

FIG. 5. (Color online) Inverted vertical profiles of the depth averaged currents using synthetic data on (a) August 24 and (b) September 11.

the range from -2.2 to $3.6 \mathrm{~cm} / \mathrm{s}$. The upper and middle layer velocities have a similar trend of variation over the period. A pseudo 10-day oscillation is apparent in the upper and middle layers, though it occurred only twice during 25 days.

In the regularization inversion, a 10-layer model is used. The thickness of the layers is set to $125 \mathrm{~m}$. Based on Eq. (8) and assuming $u_{\mathrm{ref}}=u_{\mathrm{CM}}$, the results are compared with the explicit solution in Fig. 7, after averaging over two neighboring layers for the upper two layers (0-250 and 250-500 m) and six consecutive layers for the lower layer (500-1250 m). The root mean square differences (RMSD) between the two solutions are $11.3 \mathrm{~cm} / \mathrm{s}$ for the upper layer, $3.6 \mathrm{~cm} / \mathrm{s}$ for the middle layer, and $1.6 \mathrm{~cm} / \mathrm{s}$ for the lower layer. The solution uncertainty determined from Eq. (9) is presented in the middle panel for the validity of inversion and the HYCOM currents are shown in the bottom panel for comparison. The inversion and the explicit solutions are very similar except that the vertical current shear is smaller for the inversion than for the explicit solution. The solution uncertainty varies in the range of $9-13 \mathrm{~cm} / \mathrm{s}$ for the upper layer, is $6 \mathrm{~cm} / \mathrm{s}$ for the middle layers, and is less than $10 \mathrm{~cm} / \mathrm{s}$ for the lower layers [Fig. 7(b)]. The HYCOM predictions have a temporal variation similar to the current estimates.

The vertical current profiles are constructed at depths of 125,375 , and $900 \mathrm{~m}$, corresponding to the midpoints of the respective layers (Fig. 8). The gradient of observed current is less for the inversion because of the smoothness constraint imposed in the regularization. The HYCOM predictions have the vertical profiles similar to the explicit solution, but the temporal variation of the HYCOM profiles is wider than that observed in the other solutions. The averaged shipboard ADCP profile taken on August 19 is compared with the explicit, inversion, and HYCOM profiles for August 21 (the center of 5-day smoothing window) in Fig. 8(d). A good agreement is obtained among the explicit solution, inversion, and HYCOM predictions. The ADCP data differ from the other three measurements for the following possible reasons: 
(a)

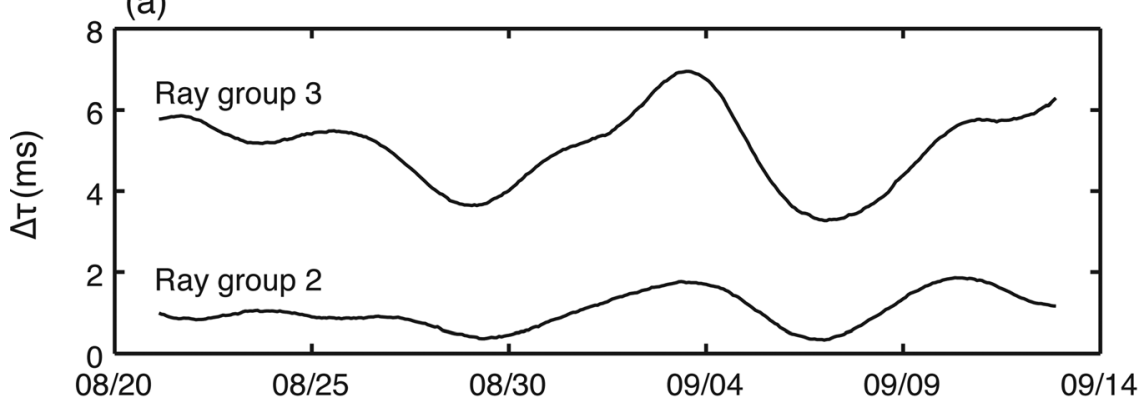

FIG. 6. Time series plots of (a) the differential travel times (after 5-day Hamming window average) and (b) the reconstructed currents from the explicit solution (solid lines for $u_{\text {ref }}=0$ and (b)

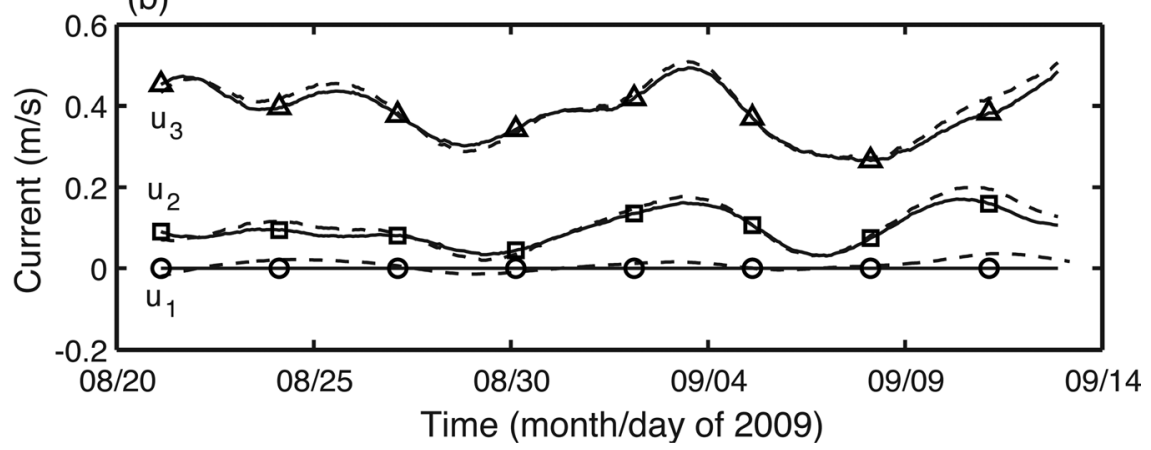

(1) They are non-simultaneous measurements with a 2 days time difference. (2) The ADCP only covers half of the upper layer, i.e., $125 \mathrm{~m}$, whereas the thickness of the layer is $250 \mathrm{~m}$. If the ADCP data were extrapolated to $250 \mathrm{~m}$ depth, the agreement would appear better.

The temporal mean and standard deviation for all the current profiles are calculated for all the three layers and listed in Table II. A good agreement of mean current is attained between the explicit solution and HYCOM predictions while the standard deviation for the HYCOM results in the middle layer is somewhat larger than for the other estimates.

\section{DISCUSSION}

Range averaged vertical current profiles in the Kuroshio southeast of Taiwan were measured using two OAT transceiver systems, located 48-km apart near the underwater sound axis (at about $1000 \mathrm{~m}$ depth). The reciprocal sound

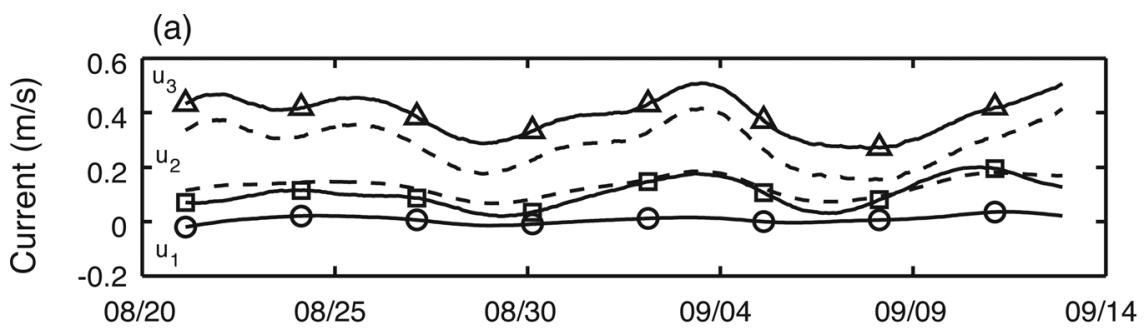

(b)

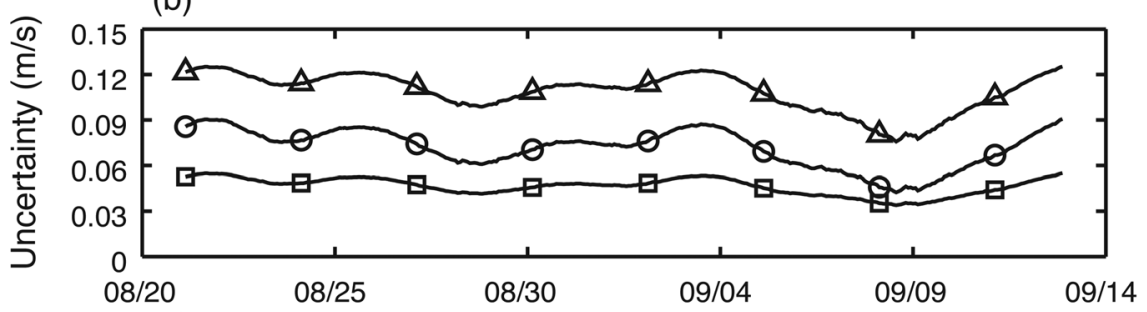

FIG. 7. (a) Comparison of the explicit solution (indicated by the solid lines) and inversion (dashed lines). Both are obtained assuming $u_{\mathrm{ref}}=u_{\mathrm{CM}}$. (b) Uncertainty estimate for inversion with regularization. (c) HYCOM predictions. The triangle, square, and circle symbols are assigned for the upper, (c)

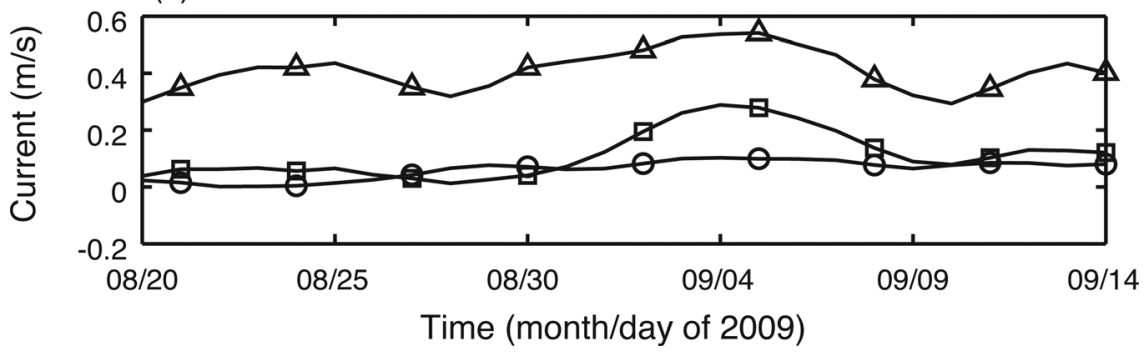
middle, and lower layers, respectively. 
(a)

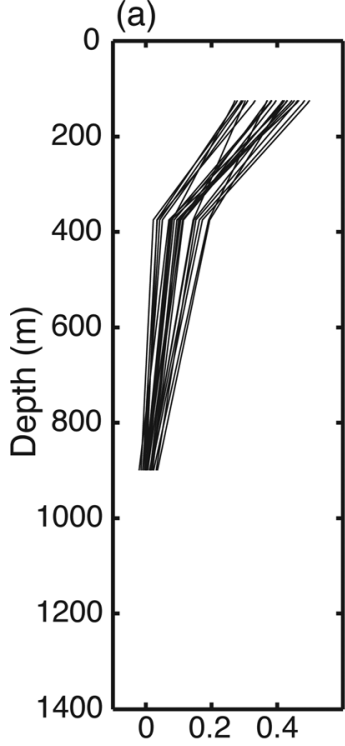

(b)

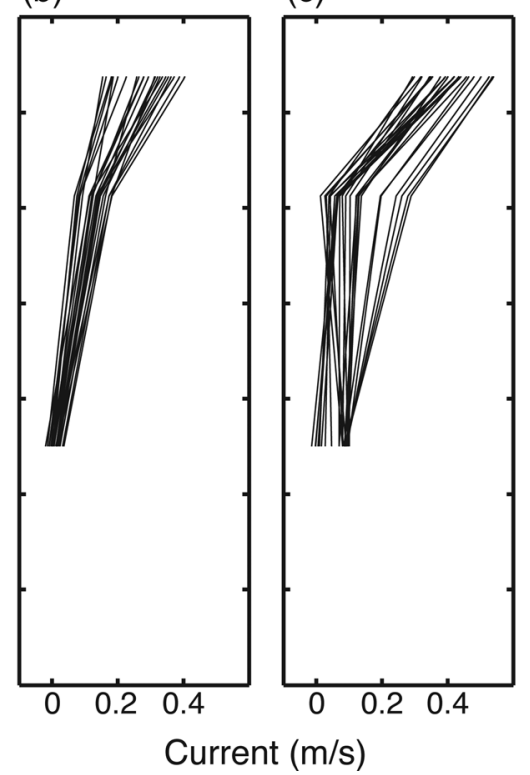

(d)

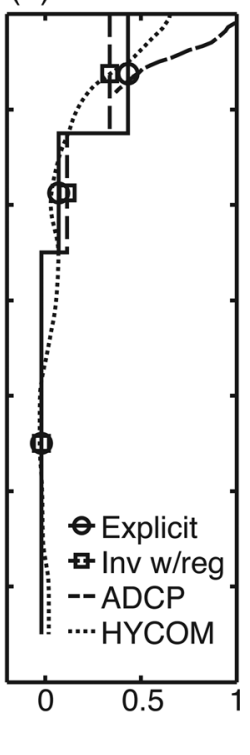

FIG. 8. Vertical profile of the depth averaged currents for (a) explicit solution, (b) inversion with regularization, and (c) HYCOM predictions, obtained during the whole observation period. (d) The estimated profiles are compared with the measurement by the shipboard ADCP for the data on August 21. transmission data were successfully obtained at a repetition interval of $1.5 \mathrm{~h}$ from August 20 to September 15, 2009. Because of a combination of factors (non-reciprocity due to bottom interaction and non-simultaneous transmissions and low SNR), conventional procedures to pick the significant arrival peaks and make arrival-peak pairs for differential travel times were not applicable, so a new method of grouping and averaging for multiple arrival peaks, the ray group method, has been developed and implemented here. Arrival peaks are divided into three ray groups by considering the depth of upper turning points of the rays. Different rays contribute to different parts of the arrival. In this environment, a later arrival usually associates with the ray having the shallower depths of upper turning point. The travel times associated with the first and last ray arrivals within each group determine the time window for the group. The differential travel times are determined as an average for each of three ray groups not by considering individual ray. Therefore the arrival time variability due to multipath interference might be reduced. The water column is also divided into three depth layers: The upper, middle, and lower layers; the latter includes the underwater sound axis. The clock error is corrected for by setting the current of the lower layer either to zero or equating it to the deep current meter measurement, which is small.

Two solution methods were used for reconstructing the vertical current profile. In the explicit solution, the number of depth layers is chosen to be equal to the number of ray

TABLE II. Comparing estimates of currents (temporal mean and standard deviation).

\begin{tabular}{lccr}
\hline \hline & \multicolumn{3}{c}{ Depth-averaged current $(\mathrm{cm} / \mathrm{s})$} \\
\cline { 2 - 4 } Layer & Explicit solution & Inversion & HYCOM \\
\hline Upper layer (0-250 m) & $39 \pm 7$ & $28 \pm 8$ & $41 \pm 7$ \\
Middle layer (250-500 m) & $10 \pm 5$ & $13 \pm 3$ & $11 \pm 8$ \\
Lower layer (500-1300 m) & $0.8 \pm 1.3$ & $0.8 \pm 1.3$ & $6 \pm 3$ \\
\hline \hline
\end{tabular}

groups. The other solution is obtained by inversion with regularization, where the current at each depth is solved for simultaneously and the number of depth layers may be greater than the number of ray groups. Both results are in good agreement. Furthermore, the HYCOM predicted current and ADCP data along the sound transmission section are used for comparison with the tomographic data. The in situ measurements - tomography and the ADCP - and the equivalent quantities derived from the HYCOM model agree within estimated uncertainties.

The pseudo 10-day period oscillation is visible in the upper two-layer results (explicit solution, inversion and HYCOM), though it occurred only twice during 25 days of measurements. It is analogous to that observed in the East Taiwan Channel (Zhang et al., 2001) and may be caused by the baroclinic instability of the Kuroshio front east of Taiwan.

\section{CONCLUDING REMARKS}

Ocean acoustic tomography is a unique tool that can make long-term measurements of overlying currents from acoustic stations located near the underwater sound axis even if the vertical resolution is limited by the ray upper turning depth. The Kuroshio flowing above a continental slope is a natural application of the method because the deployed systems are compact, near the seafloor, and robust in contrast to complicated and expensive point moorings that span the water column and are subject to trawling hazards and strong currents. Further, with now-available low power, accurate chip-scale atomic clocks, clock drift can be eliminated.

The successful use of ray groups to form differential travel times is basically an empirical result. It would be desirable to conduct thorough acoustic numerical simulations that contain the effects of eddies, advection, internal waves, and bottom bathymetry to try and reproduce both the measured arrival patterns (taking into account the $10 \mathrm{~min}$ offset) and how they were sampled/processed to obtain the average group differential travel times. 


\section{ACKNOWLEDGMENTS}

We appreciate ChangWei Lee, YenHsiang Chen and WenHwa Ho of National Taiwan University for supporting the shipboard work during the deployment and recovery of the instruments. We also thank the captain and crews of the R/V Ocean Researcher III, operated by the National Sun Yat-sen University, Taiwan. This work was supported in part by the U.S. Office of Naval Research (ONR Global/ NICOP, and Code 322 Ocean Acoustics), a grant for Frontier and Innovative Research of National Taiwan University, the National Science Council of Taiwan under NSC 99-2611-M-002-011, the National Basic Research Program of China under Grant 2011CB403503, and the Natural Science Foundation of China (41276095).

Chang, Y.-C., Tseng, R.-S., and Liu, C.-T. (2008). "Evaluation of tidal removal method using phase average technique from ADCP surveys along the Peng-Hu channel in the Taiwan Strait," Terr. Atmos. Ocean. Sci. 19, 433-443.

Chassignet, E. P., Hurlburt, H. E., Metzger, E. J., Smedstad, O. M., Cummings, J. A., Halliwell, G. R., Bleck, R., Baraille, R., Wallcraft, A. J., Lozano, C., Tolman, H. L., Srinivasan, A., Hankin, S., Cornillon, P., Weisberg, R., Barth, A., He, R., Werner, F., and Wilkin, J. (2009). "US GODAE: Global ocean prediction with the HYbrid Coordinate Ocean Model (HYCOM)," Oceanography 22, 64-75.

Cornuelle, B., Wunsch, C., Behringer, D., Birdsall, T., Brown, M., Heinmiller, R., Knox, R., Metzger, K., Munk, W., Spiesberger, J., Spindel, R., Webb, D., and Worcester, P. (1985). "Tomographic maps of the ocean mesoscale. Part 1: Pure acoustics,” J. Phys. Oceanogr. 15, 133-152.

Cornuelle, B. D., Worcester, P. F., Hildebrand, J. A., Hodgkiss, W. S., Duda, T. F., Boyd, J., Howe, B. M., Mercer, J. A., and Spindel, R. C. (1993). "Ocean acoustic tomography at 1000-km range using wavefronts measured with a large-aperture vertical array," J. Geophys. Res., [Oceans] 98, 16365-16377, doi:10.1029/93JC01246.

DeFerrari, H. A., and Nguyen, H. B. (1986). "Acoustic reciprocal transmission experiments, Florida Straits," J. Acoust. Soc. Am. 79, 299-315.

Dushaw, B., Howe, B., Cornuelle, B., Worcester, P., and Luther, D. (1995).

"Barotropic and baroclinic tides in the central North Pacific Ocean determined from long-range reciprocal acoustic transmissions," J. Phys. Oceanogr. 25, 631-647.

Dushaw, B. D., Worcester, P. F., Cornuelle, B. D., and Howe, B. M. (1994). "Barotropic currents and vorticity in the central North Pacific Ocean during summer 1987 determined from long-range reciprocal acoustic transmissions," J. Geophys. Res. 99, 3263-3272, doi:10.1029/93JC03335.

Howe, B. M., Worcester, P. F., and Spindel, R. C. (1987). "Ocean acoustic tomography: Mesoscale velocity," J. Geophys. Res. 92, 3785-3805, doi:10.1029/JC092iC04p03785.

Liu, C.-S., Liu, S.-Y., Lallemand, S. E., Lundberg, N., and Reed, D. L. (1998). "Digital elevation model offshore Taiwan and its tectonic implications," Terr. Atmos. Ocean. Sci. 9, 705-738.

Munk, W., Worcester, P., and Wunsch, C. (1995). Ocean Acoustic Tomography (Cambridge University Press, New York), Chap. 8 and Appendix A.

Park, J.-H., and Kaneko, A. (2000). "Assimilation of coastal acoustic tomography data into a barotropic ocean model," Geophys. Res. Lett. 27, 3373-3376, doi:10.1029/2000GL011600.

Porter, M. B. (2011). The BELLHOP Manual and User's Guide (Heat, Light, and Sound Research, Inc., La Jolla, CA).

Rajan, S. D., Lynch, J. F., and Frisk, G. V. (1987). "Perturbative inversion methods for obtaining bottom geoacoustic parameters in shallow water," J. Acoust. Soc. Am. 82, 998-1017.

Send, U., Schott, F., Gaillard, F., and Desaubies, Y. (1995). "Observation of a deep convection regime with acoustic tomography," J. Geophys. Res. 100, 6927-6941, doi:10.1029/94JC03311.

Taniguchi, N., Kaneko, A., Yuan, Y., Gohda, N., Chen, H., Liao, G., Yang, C., Minamidate, M., Adityawarman, Y., Zhu, X., and Lin, J. (2010). "Long-term acoustic tomography measurement of ocean currents at the northern part of the Luzon Strait," Geophys. Res. Lett. 37, L07601, doi:10.1029/2009GL042327.

Worcester, P. F., Spindel, R. C., R., and Howe, B. (1985). "Reciprocal acoustic transmissions: Instrumentation for mesoscale monitoring of ocean currents," IEEE J. Ocean. Eng. 10, 123-137.

Wunsch, C. (1996). The Ocean Circulation Inverse Problem (Cambridge University Press, New York), p. 184.

Yuan, G., Nakano, I., Fujimori, H., Nakamura, T., Kamoshida, T., and Kaya, A. (1999). "Tomographic measurements of the Kuroshio extension meander and its associated eddies," Geophys. Res. Lett. 26, 79-82, doi:10.1029/1998GL900253.

Zhang, D., Lee, T. N., Johns, W. E., Liu, C.-T., and Zantopp, R. (2001). "The Kuroshio east of Taiwan: Modes of variability and relationship to interior ocean mesoscale eddies," J. Phys. Oceanogr. 31, 1054-1074. 